\title{
LV-PP-1-4
}

\section{Eupatilin pretreatment ameliorates hepatic ischemia-reperfusion injury in mice}

\author{
Hyuk Jai JANG*, Mee Young OH
}

Department of Surgery, Gangneung Asan Hospital, University of Ulsan College of Medicine, Gangneung, Korea

Introduction: Eupatilin, a pharmacologically active flavone derived from Artemisia species, is known to have antioxidant and anti-inflammatory activities. Ischemia-reperfusion injury (IRI) is a major critical event that commonly occurs after liver transplantation and resection. Furthermore, inflammatory responses to IRI exacerbate the resultant hepatic injury. In this study, we investigated whether eupatilin protects against IR-induced acute liver injury in mice.

Methods: Partial (70\%) hepatic IRI was induced in male C57BL/6 mice by portal triad pedicle occlusion for 45 minutes followed by reperfusion for 6 hours. Eupatilin $(10 \mathrm{mg} / \mathrm{kg}$ body weight, oral) was administered 4 days before the IRI.

Results: Treatment with eupatilin significantly decreased serum alanine aminotransferase and serum aspartate aminotransferase as well as liver histological changes. Eupatilin also prevented hepatic glutathione depletion and increased malondialdehyde levels induced by IRI. Western blotting indicated that eupatilin significantly increased the levels of heat shock protein 70 and B-cell lymphoma 2 protein, attenuated inducible nitric oxide synthase, and cleaved caspase-3 levels 6 hours after IRI. The expression of the toll-like receptor $2 / 4$, and phosphorylated nuclear factor of kappa light polypeptide gene enhancer in B-cells inhibitor (p-IkB- $\alpha$ ) was significantly decreased in the eupatilin pretreatment group.

Conclusions: Eupatilin improved the acute hepatic IRI by reducing inflammation and apoptosis. These findings suggest that eupatilin is a promising therapeutic agent against acute IR-induced hepatic damage. 